\title{
Two-view Geometry Estimation Unaffected by a Dominant Plane
}

\author{
Ondřej Chum \\ Tomáš Werner \\ Jiří Matas \\ CMP, Department of Cybernetics, Czech Technical University in Prague, Czech Republic
}

\begin{abstract}
A RANSAC-based algorithm for robust estimation of epipolar geometry from point correspondences in the possible presence of a dominant scene plane is presented. The algorithm handles scenes with (i) all points in a single plane, (ii) majority of points in a single plane and the rest off the plane, (iii) no dominant plane. It is not required to know a priori which of the cases (i) - (iii) occurs.

The algorithm exploits a theorem we proved, that if five or more of seven correspondences are related by a homography then there is an epipolar geometry consistent with the seven-tuple as well as with all correspondences related by the homography. This means that a seven point sample consisting of two outliers and five inliers lying in a dominant plane produces an epipolar geometry which is wrong and yet consistent with a high number of correspondences. The theorem explains why RANSAC often fails to estimate epipolar geometry in the presence of a dominant plane.

Rather surprisingly, the theorem also implies that RANSAC-based homography estimation is faster when drawing non-minimal samples of seven correspondences than minimal samples of four correspondences.
\end{abstract}

\section{Introduction}

$T^{1} e^{1}$ topic of this paper is robust estimation of epipolar geometry (EG) from image point correspondences in the possible presence of a dominant scene plane. A novel RANSAC-based algorithm is presented that handles in a unified manner the following three classes of scenes:

1. all points belong to a single scene plane,

2. majority of points belong to a dominant plane and the rest is off the plane,

3. minority or no points lie in a scene plane (a general scene).

In the first case, only a plane homography is computed, in the other cases, a correct EG is computed. It need not be known a priori which class the input scene belongs to, and still the computations are not slower than in EG estimation by plain RANSAC for a general scene.

\footnotetext{
${ }^{1}$ The authors were supported by Czech Academy of Sciences under project 1ET101210406 and by the European Commission under project IST-004176.
}

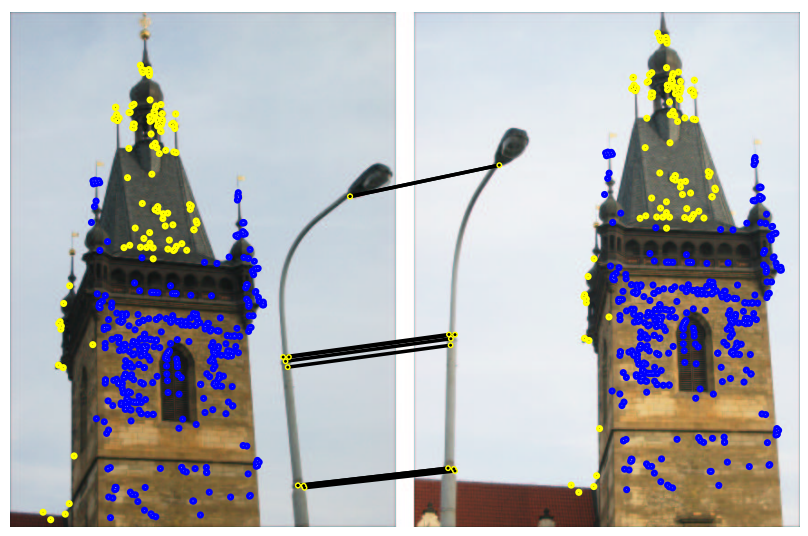

Figure 1: The LAMPPOST scene with $97 \%$ of correct tentative correspondences lying in or near a dominant plane. In 100 runs, RANSAC fails to find a single inlier on the lamp 83 times; in the remaining 17 , no more than 4 out of the 10 correspondences on the lamppost are found. Points on the lamppost are far from the dominant plane and therefore critically influence the precision of epipolar geometry and egomotion estimation. The DEGENSAC algorithm, with the same computational complexity as RANSAC, found the 10 lamppost inliers in all runs. Corresponding points lying in the dominant plane are dark, off-the-plane points are light, and the points on the lamp are highlighted by line segments.

The algorithm is based on the following theorem from two-view geometry, proved in the paper. If five or more correspondences in a sample of seven correspondences are related by a homography (we refer to such a sample as $\mathrm{H}$-degenerate), there always exists an EG consistent with both the seven correspondences and all correspondences related by the homography, including those not in the seventuple (that is, not used in the EG estimation). For example, given five correspondences related by a homography and two other arbitrary correspondences, there is an EG consistent with all correspondences related by the homography and with the two other correspondences. H-degeneracy is not necessarily indicated by ill-conditioned fundamental matrix estimation in the seven-point algorithm.

In robust EG estimation, it is standard practice to measure the quality of the EG by the number of inliers [4] or some closely related quantity such as robust likelihood [11]. It has been observed that in scenes where most points lie in a plane, standard EG estimation algorithms often return an 
EG with a high number of inliers that is however totally incorrect. This behavior is explained by the proved geometric theorem, since a high inlier count can be obtained even if the seven-point sample includes two outliers. Such a number of inliers can cause the termination of RANSAC before a non-degenerate all-inlier sample is drawn. In general, the problem cannot be overcome by ex-post local optimization such as bundle adjustment, since the solution does not include off the plane correspondences, and therefore is likely to converge to a wrong local optimum.

Exploiting properties of $\mathrm{H}$-degenerate samples, we also present a RANSAC-based algorithm for homography estimation that draws non-minimal samples. Contrary to the common practice of drawing minimal samples in RANSAC, the expected running time of the algorithm is lower than if minimal samples of four correspondences are drawn. This rather counter-intuitive result is explained by statistical analysis of the algorithm.

The planar degeneracy problem has been addressed before. In [9], different criteria for model selection are studied. The PLUNDER algorithm, which estimates multiple models separately and then performs model selection, was proposed in [8]. A method for correct feature tracking in a video sequence containing degenerate subsequences is designed in [10]. The method was extended in [7], where complete metric reconstruction is obtained. The proposed algorithm is novel since, unlike the previous approaches, it does not separately search for the two models (homography, epipolar geometry). Instead, it detects both competing models simultaneously, and the detection is not slower than direct estimation of the correct (but unknown) model.

The rest of the paper is organized as follows. In Section 2, degenerate samples are classified by the number of correspondences consistent with a homography, and properties of each class are discussed. The H-degeneracy test is developed in Section 3. A novel DEGENSAC algorithm for EG estimation unaffected by a dominant plane is presented (Section 4). Section 4.3 shows how homography can be estimated efficiently exploiting H-degeneracy. The performance of DEGENSAC is evaluated on two views of a scene with a dominant plane (Section 5). The paper is summarized and conclusions are drawn in Section 6.

\section{H-degenerate Configurations}

In the paper, symbols $\mathbf{x} \sim \mathbf{y}$ denote equality of vectors up to scale, $|\mathrm{A}|$ determinant, $[\mathbf{v}]_{\times}$cross-product matrix, and $\{x \mid \phi(x)\}$ is the set of elements $x$ with property $\phi(x)$.

Corresponding point pairs $\left(\mathbf{x}_{1}, \mathbf{x}_{1}^{\prime}\right), \ldots,\left(\mathbf{x}_{n}, \mathbf{x}_{n}^{\prime}\right)$ from two images taken by pinhole cameras satisfy the EG constraint [4]

$$
(\forall i=1, \ldots, n) \quad \mathbf{x}_{i}^{\prime \top} \mathbf{F} \mathbf{x}_{i}=0,
$$

where the $3 \times 3$ fundamental matrix $F$ has rank 2 .
Up to scale, there are 3 fundamental matrices consistent with $n=7$ correspondences in general position, of which 2 may be complex conjugates. They are computed by the 7 point algorithm [4] as follows. Matrices $F$ satisfying seven equations (1) form a 2-dimensional linear space, $\mathrm{F} \sim \lambda \mathrm{F}_{1}+$ $(1-\lambda) \mathrm{F}_{2}$. Requiring $\mathrm{F}$ to have rank 2 imposes an additional cubic constraint

$$
\left|\lambda \mathrm{F}_{1}+(1-\lambda) \mathrm{F}_{2}\right|=0
$$

A homography, represented by a $3 \times 3$ matrix $\mathrm{H}$, is said to be consistent with $\mathrm{EG}$ represented by $\mathrm{F}$ if all point pairs satisfying $\mathbf{x}^{\prime} \sim \mathrm{H} \mathbf{x}$ simultaneously satisfy $\mathbf{x}^{\prime \top} \mathbf{F x}=0$. This happens if and only if matrix $\mathrm{H}^{\top} \mathrm{F}$ is skew-symmetric [4].

The contribution of the paper is based on the following fact from geometry of two uncalibrated images. Let a set $X$ of $n$ correspondences be related by a unique homography, but otherwise in general position. If $n=7$ or $n=6$, then any EG consistent with $X$ is also consistent with the homography. If $n=5$, one of the three EGs defined by $X$ and two more correspondences is consistent with the homography. A configuration of 7 correspondences, of which 5,6 or 7 are homography related, is called an H-degenerate configuration $^{2}$.

To prove this, we start with two rather general theorems.

Theorem 1 Let $\mathrm{x}_{1}, \ldots, \mathbf{x}_{4}$ be points in a plane. The locus of such points $\mathbf{e}$ that the cross-ratio of the line pencil $\left(\mathbf{e} \times \mathbf{x}_{1}, \ldots, \mathbf{e} \times \mathbf{x}_{4}\right)$ is constant is a conic passing through $\mathbf{x}_{1}, \ldots, \mathbf{x}_{4}$.

Proof. This is a well-known theorem from the projective geometry, sometimes called Chasles' theorem [2].

Theorem 2 Let $\mathbf{x}_{1}, \ldots, \mathbf{x}_{n}$ be 5 or more points in a plane, no three of them collinear. Let $\mathrm{H}$ be a non-singular homography. The set of epipole pairs consistent with correspondences $\left\{\left(\mathbf{x}_{1}, \mathrm{Hx}_{1}\right), \ldots,\left(\mathbf{x}_{n}, \mathrm{Hx}_{n}\right)\right\}$ is given by $E=$ $E^{p} \cup E^{c}$ where

$$
\begin{aligned}
& E^{p}=\{(\mathbf{e}, \mathrm{He}) \mid \mathbf{e} \text { is arbitrary }\}, \\
& E^{c}=\left\{\left(\mathbf{e}, \mathbf{e}^{\prime}\right) \mid \text { points } \mathrm{H}_{1}, \ldots, \mathrm{H} \mathbf{x}_{n}, \mathrm{He}, \mathbf{e}^{\prime} \text { are conconic }\right\} .
\end{aligned}
$$

Proof. Assume without loss of generality $\mathrm{H}=\mathrm{I}$, that is, $\mathbf{x}_{i}^{\prime} \sim \mathbf{x}_{i}$. Then the theorem is a trivial consequence of what is called Chasles' theorem in [4, Theorem 22.3]. However, since Chasles' theorem is usually stated only for four points (Theorem 1 in this paper), we will give an explicit proof.

An epipole pair $\left(\mathbf{e}, \mathbf{e}^{\prime}\right)$ is consistent with the correspondences if line pencil $\mathbf{e}\left(\mathbf{x}_{1}, \ldots, \mathbf{x}_{n}\right)$ is projectively related to line pencil $\mathbf{e}^{\prime}\left(\mathbf{x}_{1}, \ldots, \mathbf{x}_{n}\right)$. That is, the pencil joining $\mathbf{e}$ with

\footnotetext{
${ }^{2} \mathrm{H}$-degeneracy should not be confused with the term degenerate configuration, which we use in the usual meaning to denote a configuration of 7 correspondences consistent with an infinite class of EGs.
} 
any four points of $\left\{\mathbf{x}_{1}, \ldots, \mathbf{x}_{n}\right\}$ must have the same crossratio as the pencil joining $\mathbf{e}^{\prime}$ with the same four points.

Let us choose e arbitrarily. By Theorem $1, \mathbf{e}^{\prime}$ must lie on the conic passing through points $\left\{\mathbf{x}_{1}, \ldots, \mathbf{x}_{4}, \mathbf{e}\right\}$ and, at the same time, on the conic passing through $\left\{\mathbf{x}_{2}, \ldots, \mathbf{x}_{5}, \mathbf{e}\right\}$. If these two conics are different, their common points are $\left\{\mathbf{x}_{2}, \mathbf{x}_{3}, \mathbf{x}_{4}, \mathbf{e}\right\}$. There cannot be more common points since two different non-singular conics have at most four intersections. By induction on other 4-tuples of $\left\{\mathbf{x}_{1}, \ldots, \mathbf{x}_{n}\right\}$, we obtain that either $\mathbf{e}^{\prime} \sim \mathbf{e}$ or, if points $\left\{\mathbf{x}_{1}, \ldots, \mathbf{x}_{n}\right\}$ are conconic, that $\mathbf{e}$ and $\mathbf{e}^{\prime}$ both lie on this common conic.

Theorem 2 states that a set of 6 or more homographyrelated point pairs not lying on a single conic is consistent with the same class of EGs as is the whole homography. However, if the points are conconic (which is always the case for 5 pairs), there is another class of EGs besides the one consistent with the homography, namely the class defined by epipoles lying anywhere on the respective conics.

In the theorem, the former class is denoted by $E^{p}$. This stands for the planar part of $E$, since given $\left(\mathbf{e}, \mathbf{e}^{\prime}\right) \in E^{p}$, $3 \mathrm{D}$ reconstruction of the correspondences lies in a plane. The latter part is denoted by $E^{c}$. This stands for cubic part of $E$ since the 3D reconstruction of the correspondences lies on a twisted cubic along with the two camera centers. The cubic part is closely related to the horopter theorem [4].

If the points are conconic, it can be shown that set $E$ corresponds to the set of fundamental matrices given by

$\mathcal{F}=\left\{\mathrm{F}\left|\mathrm{F}=\mathrm{H}^{-\top}\left([\mathbf{v}]_{\times}+\lambda \mathrm{Q}\right),\right| \mathrm{F} \mid=0, \mathbf{v} \in \mathbb{R}^{3}, \lambda \in \mathbb{R}\right\}$,

where matrix Q represents the Steiner conic [4, Section 9.4] passing through the points. Taking $\lambda=0$ yields the planar part $E^{p}$, whereas $\lambda \neq 0$ yields the cubic part $E^{c}$.

The rest of this section applies Theorem 2 to prove the above facts about $\mathrm{H}$-degenerate configurations.

\subsection{All 7 Pairs Related by Homography}

If all 7 out of 7 correspondences are related by a homography, the class of EGs consistent with them is the same as the class consistent with the homography. The EGs are parametrized by the position of one epipole.

Theorem 3 Let points $\mathbf{x}_{1}, \ldots, \mathbf{x}_{7}$ contain no collinear triplet and not lie on a conic. Let $\mathrm{H}$ be a non-singular homography. Then all fundamental matrices consistent with correspondences $\left\{\left(\mathbf{x}_{1}, \mathrm{Hx}_{1}\right), \ldots,\left(\mathbf{x}_{7}, \mathrm{Hx}_{7}\right)\right\}$ are consistent with $\mathrm{H}$. In detail, these fundamental matrices form a set

$$
\mathcal{F}_{7}=\left\{\left[\mathbf{e}^{\prime}\right]_{\times} \mathrm{H} \mid \mathbf{e}^{\prime} \in \mathbb{R}^{3}\right\} .
$$

Proof. Straightforward by Theorem 2. Since the points are not conconic, it is $E^{c}=\emptyset$ and $E=E^{p}$.

For this configuration, the linear space of matrices $F$ satisfying (1) has dimension 3 (rather than 2 as for a 7-tuple in a general position) and all these matrices have rank 2 . It follows that $\mathcal{F}_{7}$ is a 3 -dimensional linear space.

\subsection{6 of 7 Pairs Related by Homography}

If 6 of 7 pairs are related by a homography, the class of EGs consistent with them is the class consistent with the homography and one additional pair off the homography. The class is parametrized by the position of one epipole which has to be collinear with the last pair.

Theorem 4 Let points $\mathbf{x}_{1}, \ldots, \mathbf{x}_{6}$ contain no collinear triplet and not lie on a conic. Let $\mathrm{H}$ be a non-singular homography. All fundamental matrices consistent with correspondences $\left\{\left(\mathbf{x}_{1}, \mathrm{H} \mathbf{x}_{1}\right), \ldots,\left(\mathbf{x}_{6}, \mathrm{H} \mathbf{x}_{6}\right)\right\}$ are consistent with $\mathrm{H}$. In detail, for any $\left(\mathbf{x}_{7}, \mathbf{x}_{7}^{\prime}\right)$, fundamental matrices consistent with correspondences $\left\{\left(\mathrm{x}_{1}, \mathrm{H} \mathbf{x}_{1}\right), \ldots,\left(\mathbf{x}_{6}, \mathrm{Hx}_{6}\right)\right.$, $\left.\left(\mathbf{x}_{7}, \mathbf{x}_{7}^{\prime}\right)\right\}$ form set

$$
\mathcal{F}_{6}=\left\{\left[\mathbf{e}^{\prime}\right]_{\times} \mathrm{H} \mid \mathbf{e}^{\prime} \in \mathbb{R}^{3}, \mathbf{e}^{\prime \top}\left(\mathrm{H} \mathbf{x}_{7} \times \mathbf{x}_{7}^{\prime}\right)=0\right\} .
$$

Proof. Straightforward by applying Theorem 2 on the first 6 points. This yields $E=E^{p}$. Part of $E^{p}$ consistent with $\left(\mathbf{x}_{7}, \mathbf{x}_{7}^{\prime}\right)$ contains pairs $(\mathbf{e}, \mathrm{He})$ for $\mathbf{e}$ being collinear with the 7 th point pair.

For this configuration, the linear space of matrices $F$ satisfying (1) has dimension 2. However, unlike for a 7-tuple in a general position, all matrices in this space have rank 2. In other words, coefficients in the polynomial (2) are identically zero. Therefore, $\mathcal{F}_{6}$ is a 2 -dimensional linear space.

\subsection{5 of 7 Pairs Related by Homography}

The class of EGs consistent with the configuration of 5 homography related pairs and 2 more pairs off the homography always contains an EG consistent with the homography. This follows straightforwardly from the plane-and-parallax algorithm [5, 4], which linearly computes EG from a homography and additional 2 point pairs off the homography.

Not so obviously, this configuration is non-degenerate in the usual sense because, like a general configuration of 7 points, it yields 3 solutions for EG. In contrast, the configurations in the previous two subsections are degenerate.

By Theorem 2, the class of EGs consistent with this configuration is the same as the class consistent with a pointwise corresponding pair of conics and two additional point pairs off the homography fixed by the conics.

Theorem 6 bellow will summarize these facts. Before stating it, we give another theorem needed for its proof. It defines the class of EGs consistent with two point-wise corresponding conics and a single additional point pair (the theorem assumes $\mathrm{H}=\mathrm{I}$, hence only one conic is mentioned).

Theorem 5 Let $\mathrm{Q}$ be a conic and $\mathbf{x}$ and $\mathrm{x}^{\prime}$ two points not on $\mathrm{Q}$. Let $\left(\mathbf{e}, \mathbf{e}^{\prime}\right)$ be a pair of epipoles lying on $\mathbf{Q}$ and consistent 


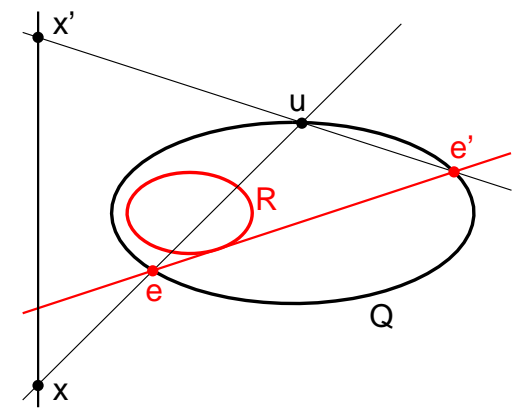

Figure 2: Conic $\mathrm{R}$ is the envelope of lines joining epipole pairs consistent with conic $\mathrm{Q}$ and the point pair $\left(\mathbf{x}, \mathbf{x}^{\prime}\right)$.

with correspondences $\{(\mathbf{y}, \mathbf{y}) \mid \mathbf{y} \in \mathbf{Q}\} \cup\left(\mathbf{x}, \mathbf{x}^{\prime}\right)$. Then the line $\mathbf{e} \times \mathbf{e}^{\prime}$ is tangent to the conic (see Figure 2)

$$
\mathrm{R}=\left(\mathbf{x}^{\top} \mathbf{Q} \mathbf{x}\right)\left(\mathbf{x}^{\prime \top} \mathbf{Q} \mathbf{x}^{\prime}\right) \mathbf{Q}-|\mathbf{Q}|\left(\mathbf{x} \times \mathbf{x}^{\prime}\right)\left(\mathbf{x} \times \mathbf{x}^{\prime}\right)^{\top} .
$$

Proof. A pair of epipoles lying on $\mathrm{Q}$ and consistent with $\{(\mathbf{y}, \mathbf{y}) \mid \mathbf{y} \in \mathbf{Q}\} \cup\left(\mathbf{x}, \mathbf{x}^{\prime}\right)$ has the property that the point $\mathbf{u}=(\mathbf{x} \times \mathbf{e}) \times\left(\mathbf{x}^{\prime} \times \mathbf{e}^{\prime}\right)$ lies on $\mathbf{Q}$. Thus, all such epipole pairs are parameterized by a point $\mathbf{u}$ moving on $\mathbf{Q}$.

Given a point $\mathbf{u}$ on $\mathbf{Q}$, the second intersection of the line $\mathbf{u} \times \mathbf{x}$ with $\mathbf{Q}$ is $\mathbf{e}=\mathbf{A} \mathbf{u}$ where $\mathrm{A}=\mathrm{I}-2 \mathbf{x x}^{\top} \mathbf{Q} /\left(\mathbf{x}^{\top} \mathbf{Q} \mathbf{x}\right)$ is the harmonic homology [4] with the vertex $\mathbf{x}$ and the axis $\mathrm{Qx}$. Similarly, $\mathbf{e}^{\prime}=\mathrm{A}^{\prime} \mathbf{u}$ where $\mathrm{A}^{\prime}=\mathrm{I}-2 \mathrm{x}^{\prime} \mathbf{x}^{\prime \top} \mathrm{Q} /\left(\mathrm{x}^{\prime \top} \mathrm{Q} \mathrm{x}^{\prime}\right)$.

If $\mathrm{Q}$ is real non-degenerate, we can without loss of generality assume $\mathbf{Q}=\operatorname{diag}(1,1,-1)$ and $\mathbf{u}=[\cos t, \sin t, 1]^{\top}$. It can be verified that the expression $\left(\mathbf{e} \times \mathbf{e}^{\prime}\right)^{\top} R^{*}\left(\mathbf{e} \times \mathbf{e}^{\prime}\right)$ vanishes identically. Hence the line $\mathbf{e} \times \mathbf{e}^{\prime}$ is tangent to $\mathrm{R}$ for any $\mathbf{u}$ lying on $\mathrm{Q}$. Here, $\mathrm{R}^{*}$ denotes the matrix of cofactors of $R$, representing the conic dual to $R$.

If the signature $\sigma$ of $\mathbf{Q}$ is different from $(1,1,-1)$, meaning than $Q$ is complex and/or degenerate, we proceed the same way using $\mathbf{Q}=\operatorname{diag}(\sigma)$ and the appropriate parameterization of $\mathbf{u}$ moving on $\mathbf{Q}$.

Alternatively, $\mathrm{R}$ is obtained by transforming $\mathrm{Q}$ by the homology [4] B with the axis $\mathbf{x} \times \mathbf{x}^{\prime}$, the vertex $\mathbf{Q x} \times \mathbf{Q \mathbf { x } ^ { \prime }}$, and the characteristic ratio $\mu=\left(\mathbf{x}^{\top} \mathbf{Q} \mathbf{x}^{\prime}\right) / \sqrt{\left(\mathbf{x}^{\top} \mathbf{Q} \mathbf{x}\right)\left(\mathbf{x}^{\prime \top} \mathbf{Q} \mathbf{x}^{\prime}\right)}$ being the projective invariant of a conic and two points [4, Exercise 2.10.2]. Unlike equation (3) however, this construction is indefinite if the line $\mathbf{x} \times \mathrm{x}^{\prime}$ is tangent to $\mathbf{Q}$.

Let $\mathbf{j}$ and $\mathbf{j}^{\prime}$ denote the common points of $\mathbf{Q}$ and the line $\mathbf{x} \times \mathbf{x}^{\prime}$, and let $\gamma$ denote the cross-ratio $\left\langle\mathbf{x}, \mathbf{x}^{\prime} ; \mathbf{j}, \mathbf{j}^{\prime}\right\rangle$. Then it can be shown that $4 \mu^{2}-2=\gamma+\gamma^{-1}$.

Assuming non-degeneracy of $Q$, conic $R$ degenerates if either $\left(\mathbf{x}^{\top} \mathbf{Q} \mathbf{x}\right)\left(\mathbf{x}^{\prime \top} \mathbf{Q} \mathbf{x}^{\prime}\right)=0$ (in that case, $R$ is the double line $\left.\mathbf{x} \times \mathbf{x}^{\prime}\right)$, or $\mathbf{x}^{\top} \mathbf{Q} \mathbf{x}^{\prime}=0$ (then homology $\mathrm{B}$ is singular and $R$ is the pair of tangents to $Q$ from the point $\left.Q \mathbf{x} \times Q x^{\prime}\right)$.

Theorem 6 Let no three of points $\mathbf{x}_{1}, \ldots, \mathbf{x}_{5}$ be collinear. Let $\mathrm{H}$ be a non-singular homography. Let $\left(\mathrm{x}_{6}, \mathrm{x}_{6}^{\prime}\right)$ and $\left(\mathbf{x}_{7}, \mathbf{x}_{7}^{\prime}\right)$ be two correspondences in general position w.r.t. the first five ones and $\mathrm{H}$. There is $s$ finite number of EGs consistent with correspondences $\left\{\left(\mathbf{x}_{1}, \mathrm{H} \mathbf{x}_{1}\right), \ldots,\left(\mathbf{x}_{5}, \mathrm{Hx}_{5}\right)\right.$, $\left.\left(\mathbf{x}_{6}, \mathbf{x}_{6}^{\prime}\right),\left(\mathbf{x}_{7}, \mathbf{x}_{7}^{\prime}\right)\right\}$ of which one EG is consistent with $\mathrm{H}$.

Proof. Assume $\mathrm{H}=\mathrm{I}$. By Theorem 2, the first five correspondences restrict the epipole pairs to be in $E^{p} \cup E^{c}$ where $E^{p}=\{(\mathbf{e}, \mathbf{e}) \mid \mathbf{e}$ arbitrary $\}$ and $E^{c}=\left\{\left(\mathbf{e}, \mathbf{e}^{\prime}\right) \mid\right.$ $\mathbf{x}_{1}, \ldots, \mathbf{x}_{5}, \mathbf{e}, \mathbf{e}^{\prime}$ conconic $\}$.

By the plane-and-parallax algorithm [4], the subset of $E^{p}$ consistent with $\left\{\left(\mathbf{x}_{6}, \mathbf{x}_{6}^{\prime}\right),\left(\mathbf{x}_{7}, \mathbf{x}_{7}^{\prime}\right)\right\}$ is given by $\mathbf{e}=$ $\left(\mathbf{x}_{6} \times \mathrm{x}_{6}^{\prime}\right) \times\left(\mathrm{x}_{7} \times \mathrm{x}_{7}^{\prime}\right)$.

Let $\mathrm{Q}$ be the conic through $\mathrm{x}_{1}, \ldots, \mathrm{x}_{5}$. By Theorem 5, the subset of $E^{c}$ consistent with $\left(\mathbf{x}_{6}, \mathbf{x}_{6}^{\prime}\right)$ is given by requiring the line $\mathbf{e} \times \mathbf{e}^{\prime}$ to be tangent to the conic which we denote by $R_{6}$. The similar conic for $\left(x_{7}, x_{7}^{\prime}\right)$ is denoted by $R_{7}$.

An epipole pair lying on $Q$ and consistent with $\left\{\left(\mathbf{x}_{6}, \mathbf{x}_{6}^{\prime}\right),\left(\mathbf{x}_{7}, \mathbf{x}_{7}^{\prime}\right)\right\}$ must lie on a common tangent to $\mathrm{R}_{6}$ and $R_{7}$. In general case, there is a finite number (up to four) of these tangents, hence there is a finite number of feasible epipole pairs and the configuration is non-degenerate.

Note, not every common tangent intersects $Q$ in a feasible epipole pair. Some do in epipole pairs consistent with $\left\{\left(\mathbf{x}_{6}, \mathbf{x}_{6}^{\prime}\right),\left(\mathbf{x}_{7}^{\prime}, \mathbf{x}_{7}\right)\right\}$ rather than with $\left\{\left(\mathbf{x}_{6}, \mathbf{x}_{6}^{\prime}\right),\left(\mathbf{x}_{7}, \mathbf{x}_{7}^{\prime}\right)\right\}$. A feasible epipole pair must satisfy that the points $\left(\mathbf{e} \times \mathbf{x}_{6}\right) \times$ $\left(\mathbf{e}^{\prime} \times \mathbf{x}_{6}^{\prime}\right)$ and $\left(\mathbf{e} \times \mathbf{x}_{7}\right) \times\left(\mathbf{e}^{\prime} \times \mathbf{x}_{7}^{\prime}\right)$ lie on $\mathbf{Q}$.

\section{Detection of H-degenerate Samples}

This section describes an efficient test whether seven correspondences $\left\{\left(\mathbf{x}_{i}, \mathbf{x}_{i}^{\prime}\right)\right\}_{i=1}^{7}$ are H-degenerate. The input is not only the seven correspondences but also a fundamental matrix F consistent with them (one of the F output by the 7-point algorithm). The test verifies whether there exist five correspondences related by a homography $\mathrm{H}$ which is consistent with $\mathrm{F}$ exist.

Given $\mathbf{F}$, only three correspondences $\left\{\left(\mathbf{x}_{i}, \mathbf{x}_{i}^{\prime}\right)\right\}_{i=1}^{3}$ are sufficient to compute a plane homography $\mathrm{H}$ as [4]

$$
\mathrm{H}=\mathrm{A}-\mathbf{e}^{\prime}\left(\mathrm{M}^{-1} \mathbf{b}\right)^{\top},
$$

where $\mathrm{A}=\left[\mathbf{e}^{\prime}\right]_{\times} \mathbf{F}$ and $\mathbf{b}$ is a 3 -vector with components

$$
b_{i}=\left(\mathbf{x}_{i}^{\prime} \times\left(\mathbf{A} \mathbf{x}_{i}\right)\right)^{\top}\left(\mathbf{x}_{i}^{\prime} \times \mathbf{e}^{\prime}\right)\left\|\mathbf{x}_{i}^{\prime} \times \mathbf{e}^{\prime}\right\|^{-2},
$$

and M is a $3 \times 3$ matrix with rows $\mathbf{x}_{i}^{\top}$.

A triplet from each five-tuple defines a homography and the other four correspondences are checked for consistency with this homography. Any of $\left(\begin{array}{l}7 \\ 5\end{array}\right)=21$ five-tuples contains at least one of the triplets $\{1,2,3\},\{4,5,6\},\{1,2,7\}$, $\{4,5,7\}$ and $\{3,6,7\}$. Hence, at most five homographies have to be tested.

In general, up to three fundamental matrices are consistent with the seven correspondences and the test should be carried for all of them. In practice, it is sufficient to check 
H-degeneracy of the F consistent with the largest number of tentative correspondences, for details see Section 4.

Note that the test is also applicable to samples containing six or seven H-related correspondences, as every fundamental matrix from the sets $\mathcal{F}_{6}$ and $\mathcal{F}_{7}$ is consistent with $\mathrm{H}$.

\section{Two-view Geometry Estimation Unaffected by a Dominant Plane}

In this section, we show how the results on degeneracies of seven point correspondences (Section 2) can be exploited to design an EG estimation algorithm that is robust to the presence of a dominant plane in the scene. Epipolar geometry estimators of practical importance must be able to handle outliers among tentative correspondences. RANSAC, which achieves robustness by drawing independent samples repeatedly, is the most commonly used EG estimator and we focus on this algorithm.

Frequently, an incorrect EG is output by RANSAC if the dominant plane is present in the scene, i.e., if a large fraction of correct tentative correspondences are related by a homography. The behavior, demonstrated in Fig. 5, is not difficult to explain. In standard RANSAC [3], it is assumed that once an all-inlier sample is drawn, the correct model is recovered. However, this is a necessary but not a sufficient condition. To estimate the EG correctly, the sample from which the EG is estimated must also be non-degenerate, i.e. at most five of its correspondences may be related by a single homography (as shown in Sections 3).

Let us analyze the RANSAC stopping criterion in detail. Let $P_{\mathrm{F}}$ be the probability that a good sample is drawn, i.e. a sample that enables estimation of the correct EG. The probability $\eta$ that RANSAC failed to find the correct solution after $k$ independent draws, i.e. no good sample has been drawn, is

$$
\eta\left(P_{\mathrm{F}}\right)=\left(1-P_{\mathrm{F}}\right)^{k} .
$$

In standard RANSAC, an all-inlier sample is assumed to be a good sample. The probability of drawing an all-inlier (seven-tuple containing seven inliers) is $P_{7 / 7}=\varepsilon^{7}$, where $\varepsilon$ is the fraction of inliers in the set of tentative correspondences. However, for the case of a dominant plane, the probabilities $P_{7 / 7}$ and $P_{\mathrm{F}}$ differ. The correct EG can only be estimated from an all-inlier sample with no more than five correspondences from the plane and 2 or more inliers off the plane. Let $\varepsilon_{\mathrm{H}}$ be the fraction of homography consistent correspondences. Then the probability $P_{\mathrm{F}}$ of drawing a good sample is

$$
P_{\mathrm{F}}=\sum_{i=0}^{5}\left(\begin{array}{l}
7 \\
i
\end{array}\right) \varepsilon_{\mathrm{H}}^{i}\left(\varepsilon-\varepsilon_{\mathrm{H}}\right)^{7-i} .
$$

Note that $P_{\mathrm{F}}=P_{7 / 7}$ if $\varepsilon_{\mathrm{H}}=0$, and $P_{\mathrm{F}}<P_{7 / 7}$ otherwise. If $\varepsilon_{\mathrm{H}}$ were known (and it never is), the probability $P_{\mathrm{F}}$ could be used to calculate the confidence $1-\eta\left(P_{\mathrm{F}}\right)$. Such a stopping rule would be very inefficient, as the $P_{\mathrm{F}}$ drops to zero when $\varepsilon_{\mathrm{H}}$ approaching $\varepsilon$.

\subsection{The DEGENSAC Algorithm}

The DEGENSAC algorithm that correctly estimates epipolar geometry even for high values of $\varepsilon_{\mathrm{H}}$ is introduced in this section. The first two steps of the algorithm, summarized in Alg. 1, are identical to standard RANSAC [4, p.291]. The algorithm repeatedly draws samples $\mathcal{S}_{k}$ of seven correspondences. The seven-point algorithm produces up to three fundamental matrices. Let $\mathrm{F}_{k}$ be the fundamental matrix with the largest support $I_{k}$ of the fundamental matrices calculated from $\mathcal{S}_{k}$. If $\mathrm{F}_{k}$ is the best so far $\left(I_{k}>I_{j}, j=\right.$ $1 \ldots k-1)$ the fundamental matrix and the size of its support is stored.

$$
\begin{array}{ll}
\text { Input: } & \text { The set of } N \text { point-to-point correspondences. } \\
\text { Output: } & \text { The fundamental matrix F with the largest sup- } \\
& \text { port; optionally the homography } \mathrm{H} \text { with the } \\
& \text { largest support. }
\end{array}
$$

Set the lower bound on the number of inliers $I^{*}:=0$.

Repeat until the probability $\eta\left(P_{7 / 7}\right)=\left(1-\left(I^{*} / N\right)^{7}\right)^{k}$ of finding EG with support larger than $I^{*}$ in $k$-th step falls under threshold $\eta_{0}$ :

1 Select a random sample of 7 correspondences, calculate up to three fundamental matrices consistent with the sample. Compute the support for each of them. Let $\mathrm{F}_{k}$ be the one with the largest support (size $I_{k}$ ). 2 If $I_{k}>I^{*}$ :

3 Store $\mathrm{F}_{k}$ and let $I^{*}:=I_{k}$.

4 Evaluate the H-degenerate sample test (see Section 3). If 5 or more correspondences from the sample consistent with homography $\mathrm{H}_{k}$ are found:

政 5 Compute the size $I_{k}^{\mathrm{H}}$ of the support of $\mathrm{H}_{k}$. Store $\mathrm{H}_{k}$ if it has the biggest support between homographies so far.

6 Execute robust estimation of fundamental matrix $\mathrm{F}_{k}^{\mathrm{H}}$ based on plane-and-parallax algorithm and calculate its support size $I_{k}^{\mathrm{F}}$.

7 If $I_{k}^{\mathrm{F}}>I_{k}$ store $\mathrm{F}_{k}^{\mathrm{H}}$ and let $I^{*}:=I_{k}^{\mathrm{F}}$.

Algorithm 1: The structure of the DEGENSAC algorithm.

When the best sample so far is drawn, the H-degeneracy test involving $\mathrm{F}_{k}$ and the seven-tuple $\mathcal{S}_{k}$ is performed (step 4) as described in Section 3. If no five correspondences are H-related, the inliers are considered to be in general position and the algorithm continues with the step 1. Otherwise, an H-degenerate sample is detected which means that a homography $\mathrm{H}_{k}$ consistent with at least five corre- 

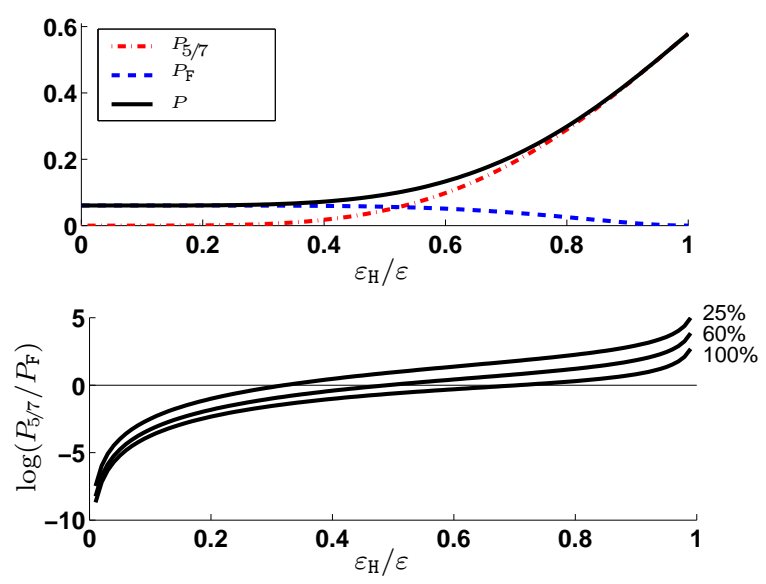

Figure 3: Probabilities $P_{5 / 7}, P_{\mathrm{F}}$ and $P$ (eqs. (6-8)), for inlier percentage $\varepsilon=67 \%$ (top) and $\log \left(P_{5 / 7} / P_{\mathrm{F}}\right)$ for values $25 \%$, $60 \%$ and $100 \%$ of $\varepsilon$ respectively (bottom).

spondences from $\mathcal{S}_{k}$ exists. Using $\mathrm{H}_{k}$, EG is estimated by the plane-and-parallax algorithm (step 6). In this case, $\mathrm{H}_{k}$ and two additional correspondences are sufficient to define an EG. The EG with the largest support found in step 6 is stored and the algorithm proceeds with the next iteration.

\subsection{DegensaC - the Probability of Success}

There are two cases how the correct EG is recovered; either from an all-inlier non-degenerate sample, or by the planeand-parallax algorithm after a homography is detected. The former case occurs with probability $P_{\mathrm{F}}$, see (6). The latter case happens when a sample containing five or more $\mathrm{H}$ related correspondences is drawn. The probability $P_{5 / 7}$ of drawing an $\mathrm{H}$-degenerate sample is

$$
P_{5 / 7}=\sum_{i=5}^{7}\left(\begin{array}{l}
7 \\
i
\end{array}\right) \varepsilon_{\mathrm{H}}^{i}\left(1-\varepsilon_{\mathrm{H}}\right)^{7-i} .
$$

The sample of five H-related and two EG consistent but not $\mathrm{H}$-related correspondences is both a good and H-degenerate sample. The probability of drawing such a sample, $21 \varepsilon_{\mathrm{H}}^{5}(\varepsilon-$ $\left.\varepsilon_{\mathrm{H}}\right)^{2}$, is included in both probabilities $P_{\mathrm{F}}$ and $P_{5 / 7}$. Therefore, following the inclusion-exclusion principle, the probability that the correct EG is recovered by drawing a single sample is

$$
P=P_{\mathrm{F}}+P_{5 / 7}-21 \varepsilon_{\mathrm{H}}^{5}\left(\varepsilon-\varepsilon_{\mathrm{H}}\right)^{2} .
$$

Note, that $P \geq P_{7 / 7}$ as an all-inliers sample is either good or H-degenerate sample, or both.

Probabilities $P_{\mathrm{F}}, P_{5 / 7}$ and $P$ are plotted in Fig. 3 (top). As $P_{\mathrm{F}}$ and $P_{5 / 7}$ are functions of both $\varepsilon$ and $\varepsilon_{\mathrm{H}}, \varepsilon=0.67$ was fixed in the figure to keep the plot one-dimensional. The figure shows, that the probability of drawing a good sample is almost unchanged up to approximately $30 \%$ of inliers lying on a single plane. The probability drops off for higher values of $\varepsilon_{\mathrm{H}} / \varepsilon$. For more than $52 \%$ of coplanar inliers, it becomes more likely to draw an H-degenerate sample than a good sample. Note that $P \geq \max \left(P_{\mathrm{F}}, P_{5 / 7}\right)$. Qualitatively, the dependency is the same for all values of $\varepsilon$. The $\log \left(P_{5 / 7} / P_{\mathrm{F}}\right)$ function for different $\varepsilon$ is drawn in Fig. 3bottom. The plot shows when it is more likely to draw a non-degenerate all-inliers sample $\left(\log \left(P_{5 / 7} / P_{\mathrm{F}}\right)<0\right)$ or Hdegenerate sample.

The termination criterion. The two algorithms, DEGENSAC and RANSAC, have equal probability of finding a correct solution after the same number of steps if $\varepsilon_{\mathrm{H}}=0$. With increasing $\varepsilon_{\mathrm{H}}$, the probability of success is increasing for DEGENSAC and decreasing for RANSAC, i.e. $\varepsilon_{\mathrm{H}}=0$ is the worst case for DEGENSAC. To ensure the required confidence in the solution even in the worst case, DEGENSAC is terminated when $\eta\left(P_{7 / 7}\right)$ falls under predefined threshold (typically 5\%).

Computational complexity. The computations carried out in DEGENSAC and RANSAC are identical with one exception - when the best sample so far is drawn. Note that other than the best samples so far are of no interest, as EG fitting as many correspondences as possible is sought for. It is not difficult to show [1], that the best sample so far occurs on average only $\log (k)$ times, where $k$ is the number of samples drawn. Thus, the procedure is executed rarely and its time complexity is not critical.

If the best sample so far is drawn, the H-degeneracy test is performed (step 4). It takes constant (and in practice insignificant) time to evaluate the test. A more complex procedure (step 6), based on the plane-and-parallax algorithm, is executed if the test detects an H-degenerate sample. In this step, another RANSAC-like strategy that draws samples of two correspondences not related by the detected homography $\mathrm{H}_{k}$ is performed. Note that the time complexity of algorithm searching for a two inlier sample is significantly lower then the complexity of the main loop.

Homography or epipolar geometry? Besides returning the EG, the DEGENSAC algorithm also outputs the homography with the largest support. The EG is valid unless a planar scene is observed or if there is a zero translation of the camera center. Some model selection strategy [9] can be applied to check the validity of the EG. The dominant plane homography may or may not be of direct interest. It can be used e.g. as a strong geometric constraint in guided matching [4].

\subsection{Homography Estimation through EG}

Consider the problem of homography estimation from data containing outliers. The standard approach (of RANSAC) is to draw samples of four correspondences to generate a hypotheses of homography. Surprisingly, a modified DE- 


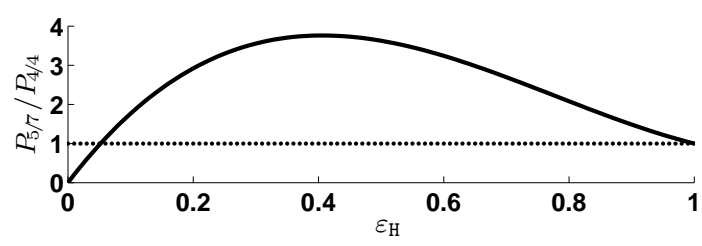

Figure 4: The ratio of probabilities of drawing an $\mathrm{H}$ degenerate sample and an all-inlier (four out of four) sample $\left(P_{5 / 7} / P_{4 / 4}\right)$.

GENSAC is almost always able to estimate the homography in a smaller number of iterations.

Instead of drawing minimal samples of four correspondences and estimating homography directly, samples of seven correspondences are drawn and epipolar geometry is estimated instead. If the support of the EG is the best so far, then the H-degenerate sample test is carried out to recover the homography. In other words, the DEGENS AC algorithm is run and the plane-and-parallax step is not executed and the estimated EG is ignored. The probability of drawing an H-degenerate sample is $P_{5 / 7}$ and therefore the algorithm is terminated when the confidence $\eta\left(P_{5 / 7}\right)$ falls under a predefined threshold.

Let us compare the probability that the solution is found in a single sample by standard RANSAC, $P_{4 / 4}=\varepsilon_{\mathrm{H}}^{4}$, and by the DEGENSAC based algorithm $P_{5 / 7}$ (7). Fig. 4 shows the ratio of the two probabilities $P_{5 / 7} / P_{4 / 4}$. For $\varepsilon_{\mathrm{H}} \in(0.052,1)$ the DEGENSAC method finds the solution in less iterations than the standard RANSAC! Note, that the range of values of $\varepsilon_{\mathrm{H}}$ where $P_{5 / 7}$ is bigger than $P_{4 / 4}$ covers all practical situations.

However, the estimation of homography through EG is unlikely to bring a significant speed-up ${ }^{3}$. Nevertheless, this is an interesting situations where a model of lower dimension can be estimated indirectly through an estimation of a model of higher dimension.

\section{Experiments}

Properties of EG estimation by the DEGENSAC and RANSAC algorithms are demonstrated on the BOX (Fig. 5) and the LAMPPOST (Fig. 1) scenes.

In the BOX experiment, both algorithms processed 958 tentative correspondences established by wide-baseline matching [6]. The set of correspondences includes 643 inliers $(\varepsilon=67 \%)$ and 613 correct matches related by a homography $\left(\varepsilon_{\mathrm{H}}=64 \%\right)$. The fraction of inliers that lie on a plane is $\varepsilon_{\mathrm{H}} / \varepsilon=95 \%$. For such values of $\varepsilon$ and $\varepsilon_{\mathrm{H}}$, the probabilities $P_{\mathrm{F}}$ of drawing a good sample (6) and a $\mathrm{H}-$

\footnotetext{
${ }^{3}$ There are up to three fundamental matrices defined by seven points and hence the decrease in the number of samples will be offset by increased number of verified fundamental matrices.
}

degenerate sample $P_{5 / 7}(7)$ are $P_{\mathrm{F}}=0.003$ and $P_{5 / 7}=0.5$ respectively.

Both the RANSAC and DEGENSAC algorithms, were executed one hundred times. The percentage of runs where a given correspondence was labeled as an inlier is plotted in Fig. 6, for both RANSAC (top) and DEGENSAC (bottom). The same correspondence occupies the same column in both plots and the correspondences are sorted according to the DEGENSAC results.

In each of the hundred execution, DEGENSAC detected the same set of $613 \mathrm{H}$-related correspondences. From the set of the remaining 345 correspondences, 329 were classified constantly in every single execution of DEGENSAC: 29 as inliers and 300 as outliers. This demonstrates the stability of the estimated EG. The remaining 16 correspondences consist of 8 correct matches and 8 mismatches that lie on the boundary of the error threshold. On average, of the 16 borderline correspondences, 4 correct matches and 2.8 mismatches were labeled as inliers per execution of DEGENSAC.

On the other hand, RANSAC often failed to find correct off-plane matches and returned an incorrect EG defined by in-plane matches and some random mismatches. To visualize the resulting epipolar geometries, four "ground truth" correspondences were established manually ex-post, two off the plane and two in the plane ${ }^{4}$.

The EG detected by DEGENSAC is shown in Fig. 5a. All four "ground truth" correspondences, both in and off the plane, satisfy the epipolar constraint. Examples of EGs estimated by RANSAC are depicted in Fig. 5b-d. Note, that even though the number of correspondences labeled as inliers in Fig. 5b-d are non random and close to the number of inliers in Fig. 5a, the resulting EG is incorrect. Only the in-plane correspondences satisfy the epipolar constraint.

Similar results were obtained for the LAMPPOST scene Fig. 1. In this experiment, the fraction of inliers was $\varepsilon=86 \%$ and the fraction of in-plane correspondences was $\varepsilon_{\mathrm{H}}=65 \% ; \varepsilon_{\mathrm{H}} / \varepsilon=76 \%$. Most of the off-plane inliers lie close to the dominant frontal plane (correspondences on the roof). Together, $97 \%$ of correspondences were in or near the plane. Ten inliers most distant from the plane, critical for the well-posedness of the solution, are located on the lamppost. In all of the one hundred DEGENSAC executions, all ten correspondences on the lamppost were labelled as inliers. RANSAC selected as inlier none of them in 83 executions. In the remaining 17 executions, RANS AC labelled no more than 4 of the correspondences as inliers.

\footnotetext{
${ }^{4}$ The manually established correspondences were, of course, not part of the input to RANSAC and DEGENSAC.
} 


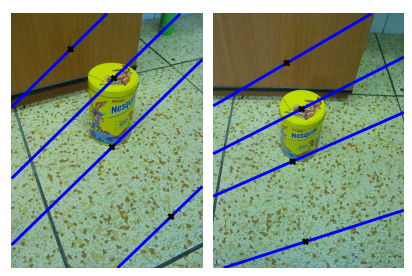

(a) 643 inliers $(\varepsilon=0.67)$

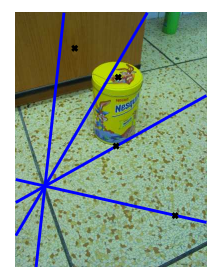

(b) 621 inliers $(\varepsilon=0.65)$

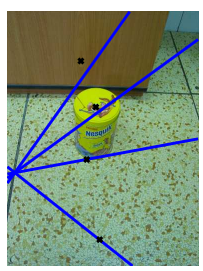

(c)

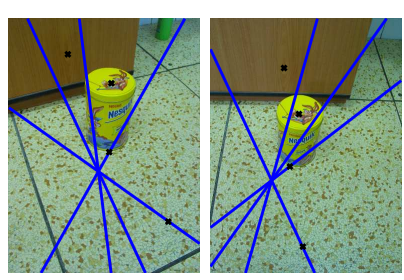

(c) 615 inliers $(\varepsilon=0.64)$
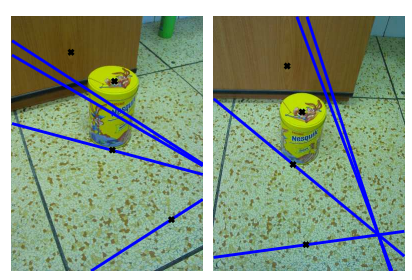

(d) 614 inliers $(\varepsilon=0.64)$

Figure 5: The BOX experiment. Examples of three EGs estimated during hundred executions of standard RANSAC (b-d); all 100 EGs estimated by DEGENSAC (a) were qualitatively the same. Epipolar lines $\mathrm{F}^{\top} \mathbf{x}^{\prime}$ and Fx for four ground truth correspondences, two off the plane and two in the plane, are superimposed over the images. The EGs on (b-d) are consistent with the floor plane, but not with the correspondences off the plane.

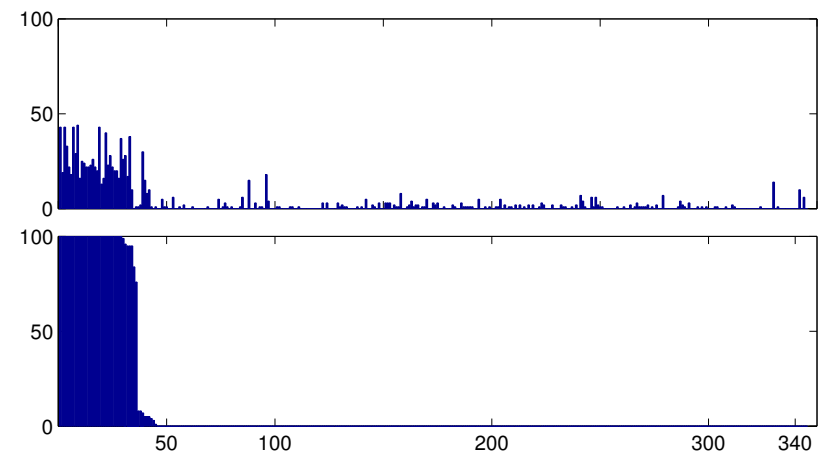

Figure 6: Each column in the graph represents a correspondence off the plane. The bars show the number of runs when the correspondence was labeled as inlier during 100 repetitions of RANSAC (top) and DEGENSAC (bottom). Note the bimodality of the DEGENSAC output.

\section{Conclusions}

The concept of H-degeneracy of a sample of seven correspondences was defined. The cases with five, six and seven $\mathrm{H}$-related correspondences were analyzed separately and a single test of H-degeneracy for all three cases was proposed.

Exploiting the results on H-degeneracy, a novel algorithm, DEGENSAC, was designed. If no large plane is present in the scene, DEGENSAC works exactly as RANSAC. If a dominant plane is detected, DEGENSAC switches to EG estimation using the plane-and-parallax strategy. The computational cost of the H-degeneracy test and, potentially, plane-and-parallax is not significant, since the steps not present in RANSAC are executed only in $\log (k)$ times, where $k$ is the total number of samples drawn.

The EG estimation process was analyzed and we showed that the larger the number of H-related correspondences, the higher the probability that DEGENS AC finds the solution. As a consequence, with the increase in the number of points in a dominant plane the running time of DEGENSAC decreases. It was demonstrated experimentally that, unlike RANSAC,
DEGENSAC estimates both the EG and the homography correctly and reliably in the presence of a dominant plane.

\section{References}

[1] O. Chum, J. Matas, and Š. Obdržálek. Enhancing ransac by generalized model optimization. In Proc. of the ACCV, volume 2, pages 812-817, January 2004.

[2] H. S. M. Coxeter. The Real Projective Plane : with an Appendix for Mathematica by George Beck. Springer-Verlag, New York, USA, 3rd edition, 1992.

[3] M. Fischler and R. Bolles. Random sample consensus: A paradigm for model fitting with applications to image analysis and automated cartography. CACM, 24(6):381-395, June 1981.

[4] R. Hartley and A. Zisserman. Multiple view geometry in computer vision. Cambridge University, Cambridge, 2nd edition, 2003.

[5] M. Irani and P. Anandan. Parallax geometry of pairs of points for 3d scene analysis. In ECCV (1), pages 17-30, 1996.

[6] J. Matas, O. Chum, M. Urban, and T. Pajdla. Robust widebaseline stereo from maximally stable extremal regions. Image and Vision Computing, 22(10):761-767, Sep 2004.

[7] M. Pollefeys, F. Verbiest, and L. van Gool. Surviving dominant planes in uncalibrated structure and motion recovery. In Proc. ECCV, volume 1, pages 837-851. Springer-Verlag, 2002.

[8] P. Torr, A. Zisserman, and S. Maybank. Robust detection of degenerate configurations while estimating the fundamental matrix. CVIU, 71(3):312-333, Sep. 1998.

[9] P. H. S. Torr. An assessment of information criteria for motion model selection. In Proc. CVPR, pages 47-53. IEEE, 1997.

[10] P. H. S. Torr, A. W. Fitzgibbon, and A. Zisserman. The problem of degeneracy in structure and motion recovery from uncalibrated image sequences. International Journal of Computer Vision, 32(1):27-44, aug 1999.

[11] P. H. S. Torr and A. Zisserman. MLESAC: A new robust estimator with application to estimating image geometry. CVIU, 78:138-156, 2000. 